\title{
CIPROFLOXACIN AND STREPTOMYCIN AS DRUGS OF CHOICE IN TREATMENT OF SURGICAL SITE INFECTIONS IN MAIDUGURI
}

\author{
${ }^{1}$ Akanmu AO, ${ }^{2}$ Anonde CE, ${ }^{1}$ Balogun ST, ${ }^{1}$ Paul LM, ${ }^{1}$ Sodipo OA
}

${ }^{1}$ Department of Clinical Pharmacology and Therapeutics, Faculty of Basic Medical Sciences, College of Medical Science, University of Maiduguri, Maiduguri, Nigeria

${ }^{2}$ Department of Medical Laboratory Science, Faculty of Allied Health Sciences, College of

Medical Sciences, University of Maiduguri, Maiduguri, Nigeria

Correspondence and reprint request to: Prof. Sulayman Tunde Balogun, Department of Clinical Pharmacology and Therapeutics, Faculty of Basic Medical Sciences, College of Medical Science,

University of Maiduguri, P.M. B.1069, Maiduguri Nigeria

Phone: +234 8065980029

\begin{abstract}
Background: Surgical site infections (SSIs) are medical events that have direct unintended consequences on patients. They often result in increased hospitalization and a greater number of diagnostic and therapeutic procedures. In Maiduguri, they are a major source of post-operative illness among hospitalized patients. Hence, it is important to determine the scope of bacteria involvement and identify suitable antibacterial agents for effective treatment. Objectives: The study was designed to determine the prevalence of SSIs in patients with surgical wounds, determine the antibiogram of the bacteria involved and drug of choice in the treatment of SSIs. Methodology: The study design was cross-sectional using the qualitative approach. Patients with surgically created wounds infected ( $>30$ days post-surgery) were enrolled between April and May 2018 at UMTH following informed consent. Swab samples were aseptically collected from the patients. The isolation and identification of bacterial isolates were done by cultural, microscopic and biochemical characterization. The antibiogram of the bacterial isolates was determined using disc diffusion method. Appropriate statistical tools were used. Results: A total of 70 patients with surgically created wounds were enrolled. The prevalence of SSIs in patients with surgically created wounds was $95.7 \%(67 / 70)$. Three bacterial species were identified withS. aureus accounting for the highest proportion of 68.7\% (46/67) while Klebsiella spp was $(19.4 \%$, $13 / 67)$ and E. coli was $(11.9 \%, 8 / 67)$ [p<0.05]. Ciprofloxacin had the highest proportion of sensitivity against S. aureus isolates with a value of $87 \%$. Streptomycin is the most sensitive drug against the Gram negative organisms isolated with a sensitivity of $87.5 \%$ and $85 \%$ for E. coli and Klebsiella spp., respectively. Conclusions: These findings suggest that there is a high prevalence of SSIs in patients with surgically created wounds seen at UMTH. Ciprofloxacin and streptomycin are suitable antibacterial agents in the treatment of the condition and this could contribute to effective treatment of patients withSSIs.
\end{abstract}

Key words: Antibiogram, Bacterial isolates, Ciprofloxacin, Streptomycin, Surgical site infections

\section{INTRODUCTION}

Surgical Site Infections (SSI) are severe events that have direct unintended consequences on patients.

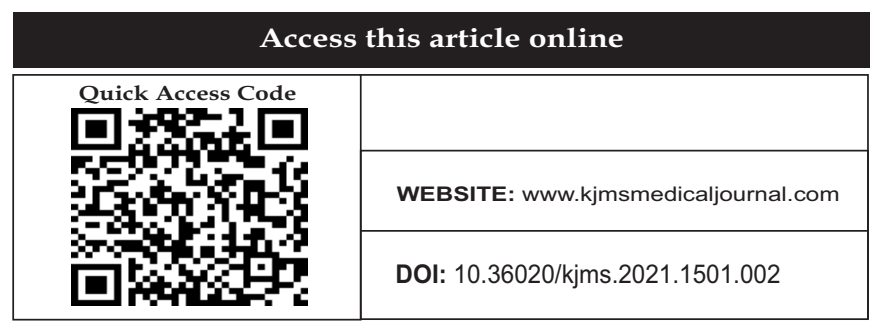

These events are directly related to an increase in hospitalisation time and a greater number of diagnostic and therapeutic procedures. "The invasion of surgical wound by bacterial pathogen within thirty days of an operation or within one year of surgery if an implant is placed in a patient is called surgical site infection". ${ }^{2}$ Surgical site infections have been reported as having the highest prevalence of all nosocomial infections recorded in orthopaedic wards. ${ }^{3}$ It is the major source of post- 
operative illness, infrequent and the highest cause of death among surgical patients in the U.S. ${ }^{4}$ Morbidity, mortality, increase in hospitalization and hospital cost is a burden to hospital resources which are caused by surgical site infection. ${ }^{5}$ SSIs are of 3 different types according to the American Center for Disease Control and Prevention (CDC) viz: superficial incisional Surgical site infections, deep incisional surgical site infections and infections involving organs or body spaces. The degree of contamination at the time of surgery influences the probability of occurrence of SSI. Surgically created wounds can also be classified as clean, clean-contaminated wounds, contaminated wounds and dirty wounds. ${ }^{6}$ The most commonly isolated organism in SSI is Staphylococcus aureus, other organisms include coagulase-negative staphylococci (CoNS), Enterococcus spp., Escherichia coli, Pseudomonas aeruginosa, and Enterobacter spp. ${ }^{7}$ Surgical procedures create widespread challenges due to undesirable events related to post-surgical infection in both developing and developed countries. ${ }^{8}$ Early treatment of SSI is an essential step in reducing its associated cost, morbidity and mortality. Gadzama et al. ${ }^{9}$ reported that Proteus spp and Pseudomonas aeruginosa were the most important gram-negative bacteria found in the isolates from infected wounds in Maiduguri. However, scientific data on the drug of choice in treatment of SSI in Maiduguri is lacking. Although, antibiotics used in preventing surgical wound infections such as: open fractures, prosthetic joint placement, puncture wounds or infected surgical wounds have long been recognized. ${ }^{10}$ However, the prevalence of antibiotic-resistant pathogens such as methicillin-resistant Staphylococcus aureus (MRSA), multi drug-resistant pathogens and others, have made the drug treatment difficult and led to an increase in the incidence of surgical site infection. ${ }^{4}$ Moreover, there is a paucity of surveillance data and scientific data in the treatment of $\mathrm{SSI}^{11}$. By the use of evidence-based drug treatment in preventing SSI and their adverse effects, one can increase patient safety, circumvent additional health care costs and ameliorate health care quality. Therefore, this study was designed to assess the prevalence of surgical site infection and serve as a guide to treat patients in surgical wards in Maiduguri.

\section{MATERIALS AND METHOD Study Area}

The study was conducted in Maiduguri, Borno State, Nigeria with an area of about $69,436 \mathrm{~km}^{2}$ located at latitude $10-30^{\circ} \mathrm{N}$ and longitude $12-15^{\circ} \mathrm{E}$. It lies within the savannah vegetation zone with low rainfall. Maiduguri is a major livestock producing area with an estimated livestock population put at $40 \%$ of national ruminant population count. ${ }^{12}$ The climate of the region is classified as hot semi-arid with a temperature peak of $44^{\circ} \mathrm{C}$ (April- May) during the hot dry season and lowest $\left(<20^{\circ} \mathrm{C}\right)$ during the dusty harmattan (December-January). It has a short rainy season (June-September) with an annual rainfall of 500-1000 mm. ${ }^{13,14}$

\section{Study Population}

The study was conducted on patients with surgically created infected wounds in the surgical wards of the University of Maiduguri Teaching Hospital (UMTH).

\section{Sample Size Determination}

The sample size for the study was determined using Thrushfield formula (2007) for random sampling ${ }^{15}$. The required sample size was thus determined (70).

Ethical Consideration and Informed Consent

Before sample collection, ethical clearance was sought from the ethical committee of the UMTH and approval was given. Patients in the surgical wards gave their consent and confidentiality of information collected was assured.

Method of Specimen Collection

Specimens were collected from surgical ward patients with surgically created wounds. The selection criteria involved wounds that were surgically created and were infected ( $>30$ days postsurgery). Wounds identified with pus draining, red and painful/hot to touch were selected and swabbed aseptically using sterile swab stick with gloved hands. Non-pus draining (uninfected) wounds were excluded. Samples were collected between April $1^{\text {st }}$ and $31^{\text {st }}$ May 2018.

\section{Sample Processing}

Swab specimens were transported immediately to the laboratory and were processed within two hours of collection.

\section{Culturing Procedure}

Each specimen on reaching the laboratory was cultured on blood, MacConkey and Chocolate agar to check for the presence of Staphylococcus species, Lactose fermenters such as Escherichia Coli and Klebsiella species as described by Carter, Quinn et al., 
Ochei and Kolhatkar. ${ }^{16-18}$ They were incubated aerobically for 24 hours at $37^{\circ} \mathrm{C}$ and the plates were examined macroscopically for colonial morphological characteristics on the following day.

\section{Biochemical Reaction and Identification}

The bacterial isolates were subjected to biochemical tests which are catalase, coagulase, indole, urea and citrate according to the description of Ochei and Kolhatkar. ${ }^{18}$

\section{Antibiotic Susceptibility Testing}

The antibiotic susceptibility testing was determined by disc diffusion method according to Clinical Stanford Laboratory Institute ${ }^{19}$ guidelines. Both gram-positive and gram-negative discs were used. Seven to eight discreet colonies of bacterial pathogens were inoculated into $5 \mathrm{ml}$ of sterile distilled water, to give fine bacterial suspensions equivalent to 0.5 in Mcfarland standard. A sterile swab was dipped into the bacterial suspension and it was used to swab the surface of the labelled Nutrient agar plate to give a confluent growth. Antibiotics discs were applied on the plate and incubated at $37^{\circ} \mathrm{C}$ for 24 hours. The zone of inhibition was measured with a standard calibrator to determine the isolate resistance or sensitivity to the applied antibiotics. ${ }^{19}$

\section{Data Analysis}

The data generated from this study were analyzed using the statistical software, Statistical Package for the Social Sciences (SPSS) version $21^{20}$. Data are expressed as mean, standard deviation and range and significance was inferred at $\mathrm{p}<0.05$.

\section{RESULTS}

Overall, 70 patients with various surgical wounds were enrolled into the study. The majority of the patients who participated in the study were males $(60.0 \%, 42 / 70)$. Out of the 70 samples collected, 67 $(95.7 \%)$ bacteria were isolated and no bacteria growth was recorded in $3(4.3 \%)$ of the total wound swabs. Table 1 shows the distribution of bacterial isolates according to the sex of the patients. Males accounted for $41(62.2 \%)$ of the samples while females accounted for $26(38.8 \%)$.

Table 2 shows the distribution of bacterial isolates recovered in surgical wound samples. Three bacterial species were identified with Staphylococcus aureus accounting for the highest proportion of $68.7 \%(46 / 67)$

Table 3 shows the antibiogram of the Staphylococcus aureus isolated from the surgical wounds of the patients. Ciprofloxacin had the highest proportion of sensitivity against the isolates with a value of 87 $\%$.

Table 4 shows the results of the antibacterial sensitivity testing for the isolated Gram negative organisms (E. coli and Klebsiella spp). Streptomycin is the most sensitive drug with a sensitivity percentage of $87.5 \%$ and $85 \%$ against E. coli and Klebsiella spp, respectively.

Table 1: Prevalence of bacterial Isolates in surgical wound samples

\begin{tabular}{lll}
\hline Bacteria isolates & Frequency & Percentage (\%) \\
\hline Esherichia coli & 8 & 11.9 \\
Klebsiella spp & 13 & 19.4 \\
Staphylococcus aureus & 46 & 68.7 \\
\hline Total & 67 & 100.0 \\
\hline
\end{tabular}

Table 2: Distribution of bacterial isolates according to sex

\begin{tabular}{llll}
\hline Bacteria isolates & Male & Female & Total \\
\hline Esherichia coli & 5 & 3 & 8 \\
Klebsiella spp & 9 & 4 & 13 \\
Staphylococcus aureus & 27 & 19 & 46 \\
Total & 41 & 26 & 67 \\
\hline Percentage (\%) & 62.2 & 38.8 & 100 \\
\hline
\end{tabular}


Table 3: Antibiotic Susceptibility pattern of the isolated S. aureus

\begin{tabular}{lllllllllll}
\hline & CPX & CH & NB & AMX & RD & GN & LEV & E & APX & S \\
\hline Sensitive \% & 87 & 54 & 52 & 65 & 63 & 59 & 63 & 57 & 54 & 65 \\
Resistance \% & 13 & 46 & 48 & 35 & 37 & 41 & 37 & 43 & 46 & 35 \\
\hline
\end{tabular}

CPX (Ciprofloxacin), CH (Chloramphenicol), NB (Norfloxacin), AMX (Amoxicillin) RD (Rifampicin), GN (Gentamycin), LEV (Levofloxacin), E (Erythromycin), APX (Ampiclox), S (Streptomycin).

Table 4: Antibiotic susceptibility pattern of Gram negative isolates

\begin{tabular}{lllllllll}
\hline $\begin{array}{l}\text { Bacteria } \\
\text { isolates }\end{array}$ & & CPX & AU & CN & OFX & S & SXT & CH \\
\hline Esherichia coli & Sensitive \% & 50 & 62.5 & 75 & 62.5 & 87.5 & 25 & 75 \\
$(\mathbf{n = 8 )}$ & Resistance \% & 50 & 37.5 & 25 & 37.5 & 12.5 & 75 & 25 \\
Klebsiella spp & Sensitive \% & 69 & 23 & 46 & 54 & 85 & 31 & 61 \\
$(\mathbf{n = 1 3})$ & Resistance \% & 31 & 77 & 54 & 46 & 15 & 69 & 39 \\
\hline
\end{tabular}

CPX (Ciprofloxacin), AU (Augumentin), CN (Gentamycin), OFX (Ofloxacin), S (Streptomycin),

SXT (Trimethoprim-sulpathoxazole), $\mathrm{CH}$ (Chloramphenicol)

\section{DISCUSSION}

Surgical site infection has posed a serious problem in hospitals for a long time and advances in the control of infections have not completely eradicated the problem. Factors that contribute to the problem include drug resistance, low level of personal hygiene and poverty. ${ }^{9}$ The present study examined the prevalence of surgical site bacterial infection among patients with surgical wounds attending UMTH and determined the antibiogram of the bacteria involved.

In this study, Staphylococcus aureus (69\%) ranked highest in occurrence compared with other bacterial isolates encountered. This finding agrees with the study conducted by Gadzama et al. ${ }^{9}$ who reported Staphyloccocus aureus as the most prevalent agent of surgical wound infections in health institutions in Maiduguri with a prevalence of $57 \%$. Therefore, this result shows that there is an increase in the proportion of wound infection caused by Staphyloccocus aureus in UMTH compared to that of Gadzama et al. ${ }^{9}$ The events leading to the infection depend on an array of factors that may be environmental or patient-related. The postulated sequence of events leading to infection could be initiated by Staphylococcus aureus nasal carriage via inhalation of infected droplets and contact with infected persons or objects which is then disseminated by the hands to other sites in the body where infection can occur through broken skin surfaces. ${ }^{21}$

The emergence of Klebsiella spp (19.4\%) and Escherichia coli (11.9 \%) as the most common Gram negative organisms in this study suggests that the isolates could be strains with high infectivity and resistance to the commonly used antibiotics in the hospital community.

The antibiogram profile of staphylococcus aureus showed that most of the strains encountered were mostly susceptible to Ciprofloxacin (87\%). This could be because Ciprofloxacin being a new generation antibiotic is not as widely abused as most of the old generation antibiotics, hence, its high degree of efficacy. However, relatively high resistance was observed for Chloramphenicol (46 $\%)$, Norfloxacin (48\%) and Ampiclox (46\%) and this could be because these drugs are often abused. In the antibiogram profile of Escherichia coli and Klebsiella spp; the organisms were $87.5 \%$ and $85 \%$ susceptible to Streptomycin respectively, followed by $75 \%$ and $61 \%$ susceptibility to Chloramphenicol and Ciprofloxacin $50 \%$ and $69 \%$ respectively, while they were highly resistant to Augmentin (77 
$\%)$, Trimethoprim-sulphamethoxazole (75\%) and Gentamycin (54\%). The rise in the antibiotic resistance noticed agrees with earlier report by Obasweiki-Ebor et al. ${ }^{21}$ where antibiotic abuse and prevalence of self-medication with antibiotics were identified as some of the factors being responsible for the antibiotic resistant bacterial strains.

Generally, Ciprofloxacin, Streptomycin and Gentamycin appeared to be much efficacious than all other antibiotics against the entire Grampositive and Gram negative isolates. This is in agreement with reports from a Tertiary care hospital in Gujarat, India. ${ }^{23}$

In conclusion, there is a high prevalence of surgical site bacterial infections in patients with surgical wound admitted in UMTH with S. aureus, Klebsiella spp. and E. coli being the bacteria of concern. The isolated $S$. aureus was largely sensitive to ciprofloxacin; the E. coli was largely sensitive to gentamicin, chloramphenicol and streptomycin. Therefore, the result of this study will assist surgical wound care practitioners in the choice of prophylactic antimicrobial agents that would prevent wound complications, increase healing rates and reduce the risk of cross-infection in health institutions in this region.

\section{Ethical Approval}

Authors hereby declared that the experimental protocol was approved by the ethical committee, University of Maiduguri Teaching Hospital and consent of patients in surgical wards was sought as well. All rules were followed as specified by national laws where applicable.

\section{Conflict of Interests}

There is no conflict of interest to declare.

\section{REFERENCES}

1. Parvizi, J., Gehrke, T. and Chen, A. F. (2013) Proceedings of the international consensus on periprosthetic joint infection. Bone joint, 95(11):1450-1452.

2. Horan, T. C., Gaynes, R. P., Martone, W. J., Jarvis, W. P. and Emori, T. G. (1992). CDC definition of Surgical Wound Infections, 13(10): 606-608.

3. Maksimović, J., Marković-Denić, L., Bumbaširević, M., Marinković, J. and Vlajinac, H. (2008). Surgical Site Infections in Orthopedic Patients: Prospective Cohort
Study. Croatian Medical Journal, 49:58-65.

4. Mangram, A. J., Horan, T. C., Pearson, M. L., Silver, L. C. and Jarvis, W. R. (1999). Guideline for Prevention of Surgical Site Infection, 1999. Centers for Disease Control and Prevention (CDC) Hospital Infection Control Practices Advisory Committee. American Journal of Infection Control, 27(2):97-134.

5. Awad, S. S., Palacio, C. H., Subramanian, A., Byers, P. A., Abraham, P. and Lewis, D. (2009). Implementation of Methicillin Resistant Staphylococcus aureus (MRSA) prevention bundle results in decreased MRSA surgical site infections. American Surgeon, 198: 607-610.

6. Di Leo, A., Piffer, S., Ricci, F., Manzi, A., Poggi, E., Porretto, V., Fambri, P., Piccini, G., Patrizia, T., Fabbri, L. and Busetti, R. (2009). Surgical site infections in an Italian surgical ward: a prospective study. Surgical Infection (Larchmt), 10(6):533-5338.

7. Zahran, W. A., Zein-Eldeen, A. A., Hamam, S. S. and Elsayed-Sabal, M. S. (2017). Surgical site infections: Problem of multidrug-resistant bacteria. Menoufia Medical Journal, 30(4):1005-1013.

8. Tariq, A., Ali, H., Zafar, F., Sial, A. A., Hameed, K. Naveed, S., Shafiq, Y., Salim, S., Mallick, N. and Hasnain, H. (2017). A Systemic Review on Surgical Site Infections: Classification, Risk Factors, Treatment Complexities, Economical and Clinical Scenarios. Journal of Bioequivalence and Bioavailability, 9(1):336-340.

9. Gadzama, G. B., Zaillani, S. B., Abubakar, D. and Bakari, A. A. (2007). Bacterial pathogens associated with wound infection at the University of Maiduguri, Nigeria. Kanem Journal of Medical Science, 1(1): 26-28.

10. Enzler, M. J., Berbari, E. and Osmon, D. R. (2011). Antimicrobial Prophylaxis in adults. Mayo Clinic Proceedings, 86(7):686-701.

11. Pathak, A., Saliba, E. A., Sharma, S., Mahadik, V. K., Shah, H. and Lundborg, C. S. (2014). Incidence and factors associated with surgical site infections in a teaching hospital in Ujjain, India. American Journal of Infection Control,42:e11-e15 
12. Gadzama, U. N., Toyosi, R. I., Madakan, S. P. and Ali, A. F. (2011). Effect of livestock, human hosts and aquatic habitat distribution on the abundance of Anopheles gambiae complex in Borno State, Arid Zone of Nigeria. International Journal of World Rural Observations, 3(4):71-77.

13. Molta, N. B., Watila, I. M., Out, T. I., Oguche, S. O., Daniel, H. I. and Gadzama, N. M. (1995). Malaria in Nigeria: an update on its chemotherapy with chloroquine, pyrimethamine/sulphadoxine and pyrimethamine/sulphalene and the need for alternative antimalarial drugs. Research Journal of Science, 1(2):59-64.

14. Gim and Associates (2002). Environmental and human resources development consultant. Environmental impact assessment of Nigerian Bottling Company PLC. Coca-cola Maiduguri Plant 'A' Wastewater discharge project: Draft report, 1-81.

15. Thrusfiled, N. (2007). Survey. In: Veterinary Epidemiology, $3^{\text {rd }}$ Edition, Blackwell Science, Oxford, UK pp 222-248.

16. Carter, G. R. (1984). Isolation and identification of bacteria from clinical specimens. Diagnostic Procedures in Veterinary Bacteriology and Mycology. 4th ed., Charles C. Thomas, USA. Pp 19-30.

17. Quinn, P. J., Carter, M. E., Markey, B. and Carter, G. R. (1994) Bacterial Pathogens: Microscopy, Culture, and Identification. In:
Clinical Veterinary Microbiology. Wolfe Publishing, London, Pp 21-80.

18. Ochei, J. and Kolhatkar, A. (2010). Medical Laboratory Science Theory and Practice. Tata McGraw-Hill Publishing Company Limited pp;537-658.

19. Clinical Laboratory and Standards Institute (CLSI) (2005). Analysis and presentation of cumulative antimicrobial susceptibility test data. Approved guideline, 2nd ed. CLSI document M39-A2, NCCLS, 940 West Valley Road, Suite 1400, Wayne Pennsylvania. pp 1809-1898.

20. SPSS (2007). Statistical Package for Social Science Windows version 16.0 SPSS Inc, Chicago, IL, USA.

21. Kluytmans, J., Van Belkum, A. and Verbrugh, H. A. (1977). Nasal carriage of Staphylococcus aureus: Epidemiology, underlying mechanisms, and associated risks. Clinical Microbiology Revision, 10: 505520.

22. Obasweki-Ebor, E. E., Akerele, J. O. and Ebea, P. O (1987). A survey of antibiotic selfmedication. Journal Antimicrobial Chemotherapy, 20:759-763.

23. Nutanbala, N. G., Hiren R. T., Alpesh P. G., Tejas K. P. and Tripathi C. B. (2011). Antibiotic sensitivity profile of bacterial pathogens in postoperative wound infections at tertiary care hospital in Gujurat, India. Journal of Pharmacology and Pharmacotherapeutics, 2: 158-164.

Cite this article as: Akanmu AO, Anonde CE, Balogun ST, Paul LM, Sodipo OA. Ciprofloxacin and Streptomycin as Drugs of Choice in Treatment of Surgical Site Infections in Maiduguri. KJMS 2021; 15(1): 13 - 18. 